\title{
Research on the Integration and Application of Abstract Elements in Freehand Oil Painting
}

\author{
Chengjian Lyu \\ Fujian Vocational College of Art, Fuzhou 350100, Fujian, China \\ Email: 1162758422@qq.com
}

\begin{abstract}
Abstraction is an indispensable element in the figurative painting. However, in the freehand oil painting which belongs to the figurative painting, the abstract elements are often covered up by the abstract language in the painting. Therefore, when many people appreciate the freehand oil painting, they can only see the artistic conception described in the painting, but they are completely unable to detect the existence of abstract elements in the painting. However, although people often ignore the appreciation of abstract elements, its existence has an important effect on freehand oil painting. It can not only enrich the content of the painting, but also enhance the connotative value of the work. Therefore, painters must pay full attention to the use of abstract elements in the freehand oil painting, so as to enrich the form and connotation of the painting and promote the effective enhancement of its artistic value.
\end{abstract}

Keywords: freehand oil painting, abstract elements, mutual integration, application value, effective measures

\section{A brief introduction of abstract elements in painting}

Chinese freehand oil painting fully inherits the freehand brushwork spirit of Chinese traditional painting view, and constantly integrates the expression of Chinese traditional freehand painting in the course of development. This kind of freehand oil painting contains abstract elements both in terms of artistic concepts and forms of artistic expression. The use of these abstract elements can not only effectively set off the main body of the painting, but also enhance the overall artistic realm of the painting and increase the artistic value of the painting. Although at present, when people recognize and appreciate freehand oil painting, they often fail to appreciate the role of abstract elements in it. Most people are confined to the artistic conception and freehand form in the painting, and completely ignore the value and role of abstract elements in the painting, but this still cannot deny the value of its existence.

In fact, Chinese people have had a good abstract aesthetic a long time ago, like the Chinese characters most familiar to Chinese people, which are typical hieroglyphs and have a very obvious abstract aesthetic tendency. As we all know, Chinese characters are a language tool that is derived from images to facilitate people's communication and exchange. Its inter-frame and form reflect the unique abstract beauty, which shows that the Chinese people had abstract aesthetic consciousness in ancient times. The pictures and symbols drawn on the clay pots and vessels used by many ancient people are also the best proof that people have abstract aesthetic consciousness.

In the traditional Chinese painting art, people pay more attention to the description of the charm of things, instead of paying too much attention to the description of the specific forms of things. However, it is worth noting that although the traditional Chinese painting art emphasizes "attaching importance to spirit and neglecting form", people have never refined the abstract elements and developed them as an independent and pure art form. This makes the traditional Chinese painting art possess the dual beauty of concreteness and abstraction, giving people unlimited visual aesthetic enjoyment, and have extremely high artistic value.

\section{The application value of abstract elements in freehand oil painting}

\subsection{Helping fully show the characteristics of Chinese oil painting}

Chinese freehand oil painting is the product of the combination of western painting art and traditional Chinese painting art. The freehand charm in traditional Chinese paintings has been completely integrated with the art form of Western oil painting. This makes Chinese freehand oil painting not only has the characteristics of Western aesthetics, but also has the details of Chinese culture, which well shows the localization characteristics of Chinese freehand oil painting. Therefore, in order to better highlight the characteristics of Chinese painting, highlight the unique charm of Chinese freehand painting, and enhance the connotation and value of Chinese freehand oil painting, the use of abstract elements cannot be ignored. ${ }^{[1]}$ People should fully realize the important role of abstract elements in freehand painting, so that the 
painting can realize the integration of concreteness and abstraction to achieve a more beautiful painting state.

\subsection{Helping strengthen the expressiveness of paintings}

Abstract element is an indispensable element in freehand oil painting. Its existence has played an important role in enhancing the expressiveness of paintings. From the point of view of the painting system, although Chinese freehand oil painting belongs to figurative painting, it has many differences from ordinary realistic painting and has the same pursuit as abstract painting in many aspects. For example, they all pay attention to the depiction of the artistic conception of the picture, and have a high aesthetic pursuit of imagery and spirit. Although the creation of realistic oil paintings is based on objectively existing objects, it does not truly and objectively portray the observed objects. Instead, it pays more attention to the description of the situation and atmosphere, pursuing a sense of beauty that blends scenes and combines nature and man.

And this artistic method itself has an extremely strong abstract aesthetic. It can be seen that incorporating abstract elements into freehand painting can greatly enhance the expressiveness of the overall picture. For a long time, the concept of "abstraction" was understood by Eastern artists as artistic conception and spirit, while in the West it was regarded as rhythm and proportion. ${ }^{[2]}$ Although people did not realize the importance of abstract elements in paintings, they have already been integrated into the art of painting and played a great role in enhancing the expressiveness of people's paintings.

\subsection{Further enriching freehand oil painting}

In fact, there are many abstract elements in traditional Chinese freehand paintings. The paintings of famous artists such as Zhu Da and Chen Zizhuang have many abstract forms of pen and ink. The concrete and abstract elements of the paintings have been well integrated, so that while the paintings fully show the meticulous and vivid scenery, they also appropriately present abstract pen and ink forms, which further enriches the overall artistic connotation of the paintings.

And it is this combination of abstraction and concreteness that enables Chinese freehand oil painting to express the "implicit and introverted beauty" in traditional Chinese culture to the fullest, which greatly enriches the art form and artistic value of Chinese freehand oil painting. Therefore, the creation of freehand oil paintings in China attaches great importance to the excavation and application of abstract elements, and only by integrating these abstract elements into freehand paintings can the art form and artistic connotation of freehand oil painting be greatly enriched.

\subsection{Effectively enhancing the aesthetic value of paintings}

Art has two sides: one is abstract and the other is concrete. Without any party, the artistic value of the work will be greatly reduced. If there are only abstract elements but no concrete elements in a painting, the whole painting will lose its substance due to the lack of concrete elements. When people watch and evaluate it, they feel as if they are in a cloud and fog, and they are confused. And if the abstract elements are missing in the painting, and only the substantive content is left, then the painting lacks charm. Not only can it not stimulate people's endless reverie, its artistic value will also be greatly reduced. When people appreciate this painting, they will only feel that "it is tasteless, and it is a pity to discard it". ${ }^{[3]}$

It can be seen from this that a painting with high aesthetic value must contain both abstract and concrete elements. Therefore, the continuous integration of abstract elements into freehand oil painting can not only better show the formal beauty of the painting, but also enhance the aesthetic value of the entire work, so that the artistic charm of freehand oil paintings can be fully displayed and expressed.

\subsection{Helping create a strong artistic atmosphere}

Only by continuously incorporating abstract elements into the creation can we create a stronger artistic atmosphere in the creation of freehand oil painting and promote the accurate expression of abstract beauty in painting. In the process of creating freehand oil painting, artists are constantly exploring and thinking with passion and pursuit for painting, wanting to break through the obstacles of appearance to find the essence of painting art and feel the abstract beauty contained in things.

In the process of their creation, they were impacted by the creative forces of reason and sensibility. This makes their painting have both a description of objective things and abstract symbols with strong subjective colors. Therefore, the artistic images created by them not only have the authenticity of objective objects, but also have artistic charm that transcends objective reality. The audience has to praise the rich artistic atmosphere that appears in the works of art. 


\section{Concrete ways to incorporate abstract elements into freehand oil painting}

\subsection{Continuously excavate and refine abstract elements}

If you want to incorporate abstract elements into the process of creating freehand oil painting, the first thing the painter has to do is to carefully observe the objective objects depicted, and then the abstract elements of the objective objects themselves from the observations, and then carry out creative activities. This requires painters to train their own observation and refinement abilities from time to time, learn how to find the unnoticeable qualities in ordinary things, and discover the artistry of things themselves. This enables themselves to fully understand and master the various relationships between things, and then integrate the abstract elements refined by thought into the creation of oil painting.

\subsection{Create with colors and shapes}

Painters can also make full use of colors and shapes to cleverly create a more ideal visual atmosphere, so that abstract elements can be better integrated into the freehand oil painting, and fully integrated with the expression of oil painting. In this way, the two complement each other and reflect each other, thereby eliminating the sense of conflict and contradiction between concrete expression and abstract elements.

Freehand oil painting is different from abstract painting. Although freehand oil painting emphasizes spirit and not form, it does not completely abandon the description of objective concrete images in terms of expression. Although it focuses more on "writing gods in form", it is very sophisticated in its depiction of the state and shape of things. Therefore, in the creation of freehand oil painting, painters should fully cultivate their own refining ability and learn to create their own unique abstract language to make it a part of their own paintings. so that artistic creation can obtain higher aesthetic value between the combination of virtual and real. ${ }^{[4]}$

\subsection{Focus on the combination of abstract elements and concrete expressions}

There are various forms and techniques of painting art creation. Many artists also have considerable differences in their painting concepts and ways of expression. Some artists may prefer to express with freehand brushwork, while others prefer abstract expressions. However, no matter what it is, the creation of freehand oil painting cannot be completely separated from the concrete painting system, regardless of whether it focuses on concrete expression or abstract expression. If the creation of freehand oil painting completely abandons the specific depiction of objective objects, it will be contrary to the artistic characteristics of freehand oil painting itself, and its appreciation value will be greatly reduced. It can be seen that only by focusing on the combination of concrete and abstract can the artistic value of freehand oil painting be effectively promoted.

\section{Conclusion}

The continuous integration of abstract elements in the creation of freehand oil painting can not only realize the perfect integration of Chinese traditional painting art and Western art, and fully demonstrate the characteristics of Chinese oil painting creation, but also further enrich the content and form of oil painting creation and create a strong artistic atmosphere. It can effectively enhance the aesthetic interest and artistic value of oil painting creation. Therefore, creators cannot ignore the role of abstract elements in the creation of freehand oil painting. Instead, they must constantly explore and think to achieve the perfect integration of abstraction and concreteness, and promote the enhancement of the artistic value of Chinese freehand oil painting.

\section{References}

[1] You Haiming. The fusion and expression of abstract elements in freehand oil painting. Artistic Sea. 2019; (12): 66-67.

[2] Chang Huaiyu. Thoughts on the "freehand brushwork" tendency in the development history of Western oil painting. Jian Nan Wen Xue (CLASSICS). 2012; (10): 176.

[3] Yao Xijuan. Research on the Aesthetic Conception of Chinese Freehand Oil Painting. Appreciation. 2018; (15): 37-38.

[4] Zhang Bin. The spiritual character of "writing" and "meaning" in Chinese freehand oil painting. Sichuan Drama. 2019; (10): 43-45. 